\title{
A Comparative Molecular Dynamics Study of Sulfuric and Methanesulfonic Acids
}

\section{Manel Canales ${ }^{\text {a) }}$ and Elvira Guàrdia}

Departament de Física, Universitat Politècnica de Catalunya, Campus Nord-Edifici B4-B5, Jordi Girona 1-3, Barcelona E-08034, Spain.

\footnotetext{
a)Author to whom correspondence should be addressed. Electronic mail: manel.canales@upc.edu
} 


\begin{abstract}
The molecular dynamics computer simulation method has been used to study sulfuric and methanesulfonic acids. Calculations have been carried out between $200 \mathrm{~K}$ and $400 \mathrm{~K}$ using reliable force fields. Thermodynamic properties, such as the density, the heat of vaporization and the melting temperature, have been computed. Moreover, structural and dynamical quantities, such as the radial distribution functions, the shear viscosity and the diffusion coefficients, have also been calculated. The results display a noticeable good agreement with the available experimental data. A hydrogen bond analysis has also been performed, which shows, on one hand, that sulfuric acid has a hydrogen bond network which resembles the one of water; and, on the other hand, that methanesulfonic acid has a hydrogen bond structure which, in some details, recalls the one of methanol, but with a more important presence of single bonds and, to a lesser extent, of branching. Finally, the dynamics of the formation and rupture of hydrogen bonds has also been analyzed. To this end, the interrupted or slow hydrogen bonding lifetimes have been calculated using two different procedures. Our findings suggest that the sulfuric acid hydrogen bond network is more labile than the methanesulfonic acid one.
\end{abstract}

\title{
KEYWORDS
}

1) Sulfuric acid.

2) Methanesulfonic acid.

3) Computer simulation.

4) Thermodynamic properties.

5) Viscosity and diffusion.

6) Hydrogen bonds. 


\section{INTRODUCTION}

Sulfuric acid $\left(\mathrm{H}_{2} \mathrm{SO}_{4}, \mathrm{SA}\right)$ is a well-known corrosive, oily, and colorless liquid at room temperature, which is widely employed in industry, especially in the production of phosphate fertilizers. It is also used in the metal processing industry, the manufacture of chemicals, paints and pigments, the petroleum refining, etc. ${ }^{1}$ Methanesulfonic acid $\left(\mathrm{CH}_{3} \mathrm{SO}_{2} \mathrm{OH}, \mathrm{MSA}\right)$, which is the simplest of the sulfonic acids, is also a colorless liquid widely used in the industry. ${ }^{2}$ Thus, it is an ideal electrolyte for many electrochemical processes because of the excellent solubility of its metal salts, the good conductivity, the low toxicity, the biodegradability and its ease of effluent treatment. ${ }^{3}$ It is also used as a catalyst in esterification and alkylation processes and to increase the conductivity of several polymers. ${ }^{4}$ MSA and SA play an important role in environmental chemistry because their aerosols influence the climate and the air visibility. ${ }^{5}$ In fact, the largest source of natural sulfur in the atmosphere is dimethyl sulfide $\left(\left(\mathrm{CH}_{3}\right)_{2} \mathrm{~S}\right)$, which is generated in the oceans by the marine phytoplankton. ${ }^{6}$ In the atmosphere dimethyl sulfide photochemically oxidizes to several sulfur compounds, such as SA and MSA; these create sulfur aerosols which act as condensation nuclei that have an important impact on the Earth climate. ${ }^{7}$

Computer simulations can be employed to check force fields by comparing the results obtained using these methods with the experimental data. So, the quality of a model will depend on the number of properties which is able to reproduce. Computer simulation studies of MSA and SA, using classical and quantum mechanical methodologies, have been fulfilled in the last twenty years. Then, ab initio methods have been used to calculate the structure, the formation energy of small SA hydrates and the minimum number of water molecules to protonate SA molecules. ${ }^{8}$ Monte Carlo simulations, with a constant ${ }^{9}$ and a fluctuating ${ }^{10}$ number of water molecules, have also been carried out to study the nucleation process of the SA-water system, providing interesting results on the shape, the free energy and the 
dissociation of the clusters. The molecular dynamics (MD) methodology has also been used to address similar topics. ${ }^{11}$ In addition, it has been applied to analyze the destabilization of the clusters structure associated to the protonation of the acid molecules, ${ }^{12}$ and to study the increasing of the nucleation process with the SA concentration. ${ }^{13}$ Also, $a b$ initio $^{14}$ and classical $^{15} \mathrm{MD}$ simulations of aqueous SA solutions at several concentrations have been carried out to analyze structural and interfacial properties. These studies have shown the reliability of several force fields, which in much cases, incorporates the protonation of the SA molecules. Some $a b$ initio studies of pure MSA have been implemented to calculate enthalpies of formation, structures and spectroscopic properties. ${ }^{16-18}$ The same methodology has been used to analyze the structure and the energy of hydrated MSA clusters, and to investigate the influence of the hydrogen bond network in the polarizabilities of the molecules. ${ }^{19-21}$ Finally, MD simulations of pure MSA $^{22}$ and MSA-water mixtures ${ }^{23}$ have also been performed.

Hydrogen bonds (HB) are present in a large number of biological and chemical systems. The computation of the mean number of HB per molecule and their lifetime is very important to understand many details related to the structure and dynamics of these systems. ${ }^{24,25}$ Simulations of water and other hydrogen bonded liquids, such as alcohols, have been carried out during the last forty years. ${ }^{26}$ It is well-known that water shows a topologically complex three dimensional tetrahedral network of $\mathrm{HB}^{27}$ On the contrary, as methanol molecules have one HB donor and a methyl group, a series of linear winding chains with several branches dominates the topology of liquid methanol ${ }^{28}$. These branched structures (i.e. the so called branching) are originated from molecules with more than 2 HB. Otherwise, every SA and MSA molecule has two types of oxygen atoms: in one hand, the oxygen atoms of the sulfonyl group (two in both cases), which are bonded to the sulfur atom by a double bond, and, on the other hand, the oxygen atoms of the hydroxyl group (one for MSA and two for SA), that are 
bonded to the hydrogen and sulfur atoms by single bonds, which act as HB donors. Therefore, every MSA and SA molecule has, respectively, one and two HB donors.

Recently, Canales and Aleman ${ }^{22}$ have carried out a MD study of pure MSA. Their objectives were, in one hand, to look for a force field which reasonably reproduces a set of experimental data and, on the other hand, to study its HB structure. They concluded that only the sulfonyl oxygen atoms act as HB acceptors. Moreover, they also stated that the HB network resembles the one of methanol, but with some branching and single hydrogen bonding. So, the existence of one donor and two acceptors per molecule gives rise to a particular HB structure which, in some aspects, resembles the one of methanol.

The main objectives of the present work are, in one hand, to find reliable force fields for liquid SA and MSA allowing to reproduce the maximum number of experimental data and, on the other hand, to compare both systems, at the atomistic scale, in a large range of temperatures. The ultimate goal is the computation of the activation energies and the calculation of the mean number of $\mathrm{HB}$, and their lifetimes, analyzing the influence of the presence of one (in case of MSA) and two (in case of SA) donors per molecule. The paper is organized as follows: Simulation details are explained in the following section, the Results and Discussion section is devoted to the exposition of the structural, dynamical and thermodynamic properties, and also the HB analyses. Finally, the concluding remarks are summarized in the last section.

\section{METHODOLOGY}

\section{A. Ab initio partial charges and geometries}

The SA and MSA partial charges and their equilibrium geometry have been obtained using the Gaussian 03 program. ${ }^{29}$ Specifically, the bond lengths, the bending bond angles and the torsional dihedral angles have been calculated. The quantum-mechanical computations have been made at the density functional level of theory (DFT) using the Becke's three- 
parameter exchange hybrid functional (B3) ${ }^{30}$ and the nonlocal correlation functional of Lee, Yang and Parr (LYP). ${ }^{31}$ This exchange-correlation functional (B3LYP) was combined with the aug-cc-PVDZ basis set. The atomic charges have been determined by fitting them to match the molecular electrostatic potential using the Merz-Singh-Kollman scheme. ${ }^{32,33}$ These charge values and those of Artsila et al., ${ }^{8}$ Ding et al. ${ }^{11}$ and Loukonen et al. ${ }^{34}$ are gathered in Table I. Our results are in a good agreement with those computed by Loukonen et al. ${ }^{34}$ in their study of the hydration of SA - Ammonia and SA - Dimethylamine clusters. Finally, it should be noted that the aug-cc-PVDZ basis set has been used because the computed geometric parameters are very similar to the available experimental results and, at the same time, because the charge values are smaller than those obtained with simpler basis sets.

\section{B. Force fields and computer simulation details}

MD simulations have been performed using the GROMACS 4.6 package. ${ }^{35}$ Bond lengths have been constrained using the SHAKE algorithm. ${ }^{36}$ The bond bending angle interactions have been computed using the OPLS harmonic angle functional force field. ${ }^{37}$ The bond lengths and the bending angles are those obtained from our DFT calculations. In most cases the OPLS-AA bending force parameters ${ }^{37}$ have been considered. However, the bending interactions involving $\mathrm{S}$ atoms have been computed using the parameters recently proposed by Hetko et $a l^{38}$ in their computer simulation study of sulfur dioxide. The torsional potentials, referred to the following dihedrals, have been computed: H-C-S-O,O’ (MSA), H'O'-S-O,C (MSA) and H'-O'-S-O,O' (SA). The first one shows three clear minima located at $-60,60$ and $180^{\circ}$ and the two others at $-120,0$ and $120^{\circ}$. They have been modeled using the formalism proposed by Ryckaert and Bellemans. ${ }^{39}$ The parameters of the bonding, bending and torsional interactions are collected in Tables II, III and IV. The Lennard-Jones 12-6 potential has been employed to model the van der Waals interactions. The homoatomic pair parameters of the OPLS-AA force field, ${ }^{35}$ which are gathered in Table V, have been used. 
The corresponding heteroatomic pair parameters are obtained, from the homoatomic ones, using the Lorentz Berthelot mixing rules. The electrostatic contributions have been calculated using the set of partial charges gathered in Table V. These are very similar to those employed by Artsila et al. ${ }^{8}$, Ding et $a l^{11}$ and Paddison ${ }^{40}$ to study several sulfuric acid-water and sulfonic acids-water systems. It is important to noticed that these charge values are smaller than the ones computed by ourselves and those proposed by Loukonen et al. ${ }^{34}$ (see Table I). We have not employed these values because the viscosity coefficient results obtained using them are clearly much larger than the experimental data, in spite of the fact that the structural properties and the density are in a reasonable good agreement with the available experimental results. Finally, it should also be remarked that the force field is different to that utilized in our previous MSA study. ${ }^{22}$ In that case the GROMOS model ${ }^{41}$ was employed, with the bond stretching and the bending terms modeled using, respectively, fourth power and cosine based angle potentials. Moreover, the parameters of the torsional potential and the larger partial charge values of the $\mathrm{CH}_{3}$ group, employed in the present work, are more suitable to keep every MSA molecule close to its equilibrium geometry.

MSA and SA have been simulated using a set of 500 molecules in the isothermal-isobaric (NPT) ensemble at $298 \mathrm{~K}$ and $1 \mathrm{~atm}$ with an integration time step of $1 \mathrm{fs}$. The Berendsen weak coupling temperature and pressure bath methods ${ }^{42}$ have been employed to control the temperature and the pressure. To this end, the time constant of the heat bath coupling and the pressure relaxation time were, respectively, 0.1 and 1 ps. The ordinary periodic boundary conditions have been considered. A cutoff distance of $1 \mathrm{~nm}$ has been used to truncate the Lennard-Jones potential. The well-known Ewald summation method ${ }^{43}$ has been utilized to compute the electrostatic interactions. In this case, the real space term was truncated at the van der Waals distance cut-off. The reciprocal space contribution has been determined by interpolation into an infinite grid of points (Particle Mesh Ewald), ${ }^{44}$ the grid spacing being 
$0.12 \mathrm{~nm}$. The following procedure has been applied to get the equilibrated starting structure of every system: a first configuration has been created introducing at random 500 molecules inside a cubic box with a length of $4 \mathrm{~nm}$. Later, the system has been relaxed after $10^{4}$ steps of energy minimization, using a steepest descent algorithm. ${ }^{45}$ Finally, 10 ns NPT-MD configurations have been generated to definitively equilibrate the system. The properties have been computed from $10^{7}$ NPT-MD configurations. In all cases the coordinates and velocities have being saved every 100 steps. To study the temperature dependence of some properties, a set of 21 NPT-MD simulations, in the range between 200 and $400 \mathrm{~K}$ with a temperature step of $10 \mathrm{~K}$, but in all cases at $1 \mathrm{~atm}$, have been carried out.

\section{RESULTS AND DISCUSSION}

\section{A. Thermodynamic and structural properties}

The bond lengths and the bending angles of both molecules, obtained from our DFT calculations, are displayed in Tables II and III. For MSA, these results agree well with the structural parameters previously obtained by other authors. ${ }^{16-18,34}$ It is important to notice, that according to Durig et al., ${ }^{16}$ we have analyzed the gauche $\mathrm{OH}$ group configuration. In this geometric arrangement, which correspond to a local minimum, the hydrogen atom quasieclipses one of the oxygen atoms. Moreover, according to these authors, ${ }^{16-18}$ the $\mathrm{CH}_{3}$ group conformation is staggered. For SA the simulation results show a good agreement with the experimental findings of Kuczkoswki et al. ${ }^{46}$ (see Fig. 1 of this reference) and Givan et al. ${ }^{47}$, and also with the $a b$ initio study of Arstila et $a l^{8}$ A plot of the most favorable structures of both molecules is depicted in Fig. 1.

The MD density values at $298 \mathrm{~K}$ and $1 \mathrm{~atm}$ are $1474 \mathrm{~kg} / \mathrm{m}^{3}$ (MSA) and $1859 \mathrm{~kg} / \mathrm{m}^{3}$ (SA), which agree well with the experimental data, ${ }^{48,49}$ at the same temperature and pressure, 1475 $\mathrm{kg} / \mathrm{m}^{3}$ and $1826 \mathrm{~kg} / \mathrm{m}^{3}$. 
The thermal expansion coefficient of both systems has been determined analyzing the temperature dependence of the density which has been plotted in Fig. 2. A sound linear behavior, between 300 and $400 \mathrm{~K}$, is observed. A least squares fit yields the following values: $80.4 \times 10^{-5} \mathrm{~K}^{-1}$ (MSA) and $67.2 \times 10^{-5} \mathrm{~K}^{-1}$ (SA). In this last case, it should be noted that the simulation result is slightly larger than the experimental value: ${ }^{50} 56 \times 10^{-5} \mathrm{~K}^{-1}$. Unfortunately, to our knowledge, for MSA no experimental data can be found in the literature.

The intermolecular structure of liquids is generally characterized by the center of masscenter of mass $g_{C M-C M}(r)$ and the partial radial distribution functions, such as the hydrogenoxygen $g_{H-O}(r)$ and the oxygen-oxygen $g_{O-O}(r)$. It should be noted that the center of mass for both molecules is almost located at the position of the sulfur atom. The $g_{C M-C M}(r)$ calculated at 200, 298 and $400 \mathrm{~K}$ have been displayed in Fig. 3 (top panel: MSA left and SA right Both acids show similar distribution functions with three clear peaks, located at $0.48 \mathrm{~nm}, 0.95 \mathrm{~nm}$ and $1.35 \mathrm{~nm}$. It should be noticed that the first peak is placed at a distance very close to the one deduced by Andreani et $a l .{ }^{51}$ from X-ray and neutron diffraction experiments. Particularly, these authors stated that the average distance between sulfuric acid molecules is approximately $0.53 \mathrm{~nm}$. Moreover, the positions of the maxima and minima do not significantly change with temperature, which only slightly affects the intensity of the first maximum. In both cases the first complete shell has 12 neighbor molecules. The orientational correlation functions $<\cos \theta(r)>$, where $\theta(r)$ is the angle between the dipole moments of two molecules whose center of masses are separated a distance $r$, are also depicted in Fig. 3 (bottom panel). For both systems, near neighboring molecules clearly exhibit an antiparallel dipole-dipole alignment. This behavior weakens but still persists at larger molecule-molecule separations. Also note that the orientational correlations are more marked for MSA than for SA. This probably happens as a consequence of the lower symmetry of the MSA molecule, just because it has a methyl group where the SA molecule has a hydroxyl group (see Fig. 1). 
The $g_{H-O}(r)$ and the contributions related to the oxygen atoms of the sulfonyl $(\mathrm{S}=\mathrm{O})$ and hydroxyl (S-O-H) groups at $298 \mathrm{~K}$ are plotted in Fig. 4. Both systems exhibit similar results with two clear peaks, located at $0.18 \mathrm{~nm}$ and $0.41 \mathrm{~nm}$, and two minima at $0.25 \mathrm{~nm}$ and 0.47 nm. It should be noted that the first peak is located at the same hydrogen bonding distance $r_{H O}$, computed by Durig et al. ${ }^{16}\left(r_{H O}=0.1784 \mathrm{~nm}\right.$, for MSA) and Kazansky and $\operatorname{Solkan}^{52}\left(r_{H O}\right.$ $=0.1809 \mathrm{~nm}$, for SA) in their ab initio studies of MSA and SA dimers. Moreover, the most important contribution to the radial distribution function at short distances $(<0.3 \mathrm{~nm})$ is mainly due to the oxygen atoms of the sulfonyl group. This result indicates that these oxygen atoms are the hydrogen bond acceptors. The partial $g_{H-O}(r)$ functions calculated at $200 \mathrm{~K}, 298$ $\mathrm{K}$ and $400 \mathrm{~K}$ have been plotted in Fig. 5 (top). Again, the temperature does not significantly change the locations of the maxima and minima. However, the first peak intensity clearly decreases when the temperature rises. Finally, the $g_{O-O}(r)$ functions of both systems at $298 \mathrm{~K}$ have been plotted in Fig. 5 (bottom). Two maxima, located at $0.31 \mathrm{~nm}$ and $0.52 \mathrm{~nm}$, and two minima, at $0.4 \mathrm{~nm}$ and $0.65 \mathrm{~nm}$, are observed.

The methodology of Caleman et $a .^{53}$ has been employed to calculate the enthalpy of vaporization:

$$
\Delta H_{\text {vap }}=\left[E_{\text {pot }}(g)+k_{B} T\right]-E_{\text {pot }}(l)
$$

$E_{p o t}(g)$ and $E_{p o t}(l)$ are respectively the potential energy of the gas and liquid phases. $E_{p o t}(g)$ has been obtained running a simulation of a single molecule using the GROMACS stochastic dynamics integrator, ${ }^{35}$ which basically consists on the solution of the Langevin equation of motion:

$$
m_{i} d^{2} \boldsymbol{r}_{i} / d t^{2}=-m_{i} \xi_{i} d r_{i} / d t+F_{i}(r)+\rho_{i}(t)
$$

$m_{i}$ being the mass of atom $i, \xi_{i}$ a friction constant and $\rho_{i}(t)$ a random force with

$$
<\rho_{i}(t) \rho_{j}(t+s)>=2 m_{i} \xi_{i} k_{B} T \delta(s) \delta_{i j}
$$


where $k_{B}$ is the Boltzmann's constant and $T$ the temperature. $\delta(s)$ and $\delta_{i j}$ are respectively the Dirac and the Kronecker delta functions. $F_{i}(r)$ is the term associated to the force field, which in this case only has intramolecular contributions. After equilibration, the gas phase potential energy has been calculated from a 1 ns simulation. The heat of vaporization of MSA at $298 \mathrm{~K}$ is $66.8 \mathrm{~kJ} / \mathrm{mol}$. This result is in a reasonable good agreement with the experimental data 75.19 $\mathrm{kJ} / \mathrm{mol}$ determined by Guthrie et $a l .{ }^{54}$ For SA the experimental value, ${ }^{1}$ referred to its boiling point $(603 \mathrm{~K})$, is $56 \mathrm{~kJ} / \mathrm{mol}$, which is in a good agreement with our simulation result at this temperature, which yields $53.03 \mathrm{~kJ} / \mathrm{mol}$.

Finally, it is important to point out that, for MSA, the thermodynamic and structural results are very close to those obtained in our previous work. ${ }^{22}$

\section{B. Viscosity and diffusion}

As it is well known viscosities can be computed from MD simulations. ${ }^{43}$ Then, in one hand, equilibrium MD simulations can be used to calculate the non-diagonal terms of the stress tensor and their correlation functions; so, in this case, the shear viscosity is obtained using the Green-Kubo or the Einstein relations. On the other hand, it can also be achieved

from non-equilibrium MD simulations. In this way, some years ago $\mathrm{Hess}^{55}$ made a comparative study of many of these methodologies for water and several Lennard-Jones fluids. He concluded that the one based on the periodic perturbation method was the most efficient. In this case the system is placed in a conventional simulation box, but a periodic external force is applied to all particles in one direction (say $x$ ) which varies in a perpendicular direction (say z). Then, a velocity field is created which verifies the NavierStokes relation, the viscosity being directly related to the velocity profile. This relationship strongly depends on the amplitude $\lambda$ of the acceleration. So, in one hand, $\lambda$ should be small enough so that the perturbation does not significantly modify the equilibrium condition and, on the other hand, small forces induce to low signal-to-noise ratio. The key of the method is 
to use a set of small $\lambda$ values and to extrapolate the results to zero by a linear fit. Our findings are summarized in Fig. 6. The MSA and SA shear viscosity coefficients are respectively $11.6+/-0.2 \mathrm{cP}$ and $21.9+/-0.3 \mathrm{cP}$, which are in a good agreement with the experimental data $^{48,56} 11.21 \mathrm{cP}$ and $23.8 \mathrm{cP}$.

The self-diffusion coefficient $D$ has been obtained, by means of the Einstein relation, ${ }^{43}$ from the long-time slope of the mean square displacements of the center of mass molecules. The simulation results at room temperature for MSA and SA are, respectively, $(109.1 \pm 5.1) \times 10^{-12} \mathrm{~m}^{2} / \mathrm{s}$ and $(60.9 \pm 3.7) \times 10^{-12} \mathrm{~m}^{2} / \mathrm{s}$. This last value agrees well with the experimental result ${ }^{57}$ determined by Harris $65.7 \times 10^{-12} \mathrm{~m}^{2} / \mathrm{s}$. Although to our knowledge there is not experimental data for MSA, it is possible to check the simulation value using the Stokes-Einstein relation formula. ${ }^{58}$ This equation relates the diffusion coefficient $D$ of a spherical "Brownian particle" of diameter $d$ to the shear viscosity $\eta$ coefficient of the fluid where the particle is immersed:

$$
D=k_{B} T /(q d \pi \eta)
$$

$k_{B}$ being the Boltzmann constant, $T$ the temperature and $q$ a coefficient which depends on the boundary conditions at the particle-fluid interface. $q=2$ and 3, respectively, for the slip and stick boundary conditions. Although this relation refers to a "Brownian particle" in a fluid, it often works reasonably well if the sizes of the particle and the solvent molecules are similar and, particularly, when the slip condition $(q=2)$ is assumed ${ }^{59}$. The diameter of the MSA and SA molecules can be evaluated taking into account that the first maximum of the $g_{C M-C M}(r)$ functions, plotted in Fig. 3, is located at $0.48 \mathrm{~nm}$. Then, the $q$ factor computed from equation 5 at room temperature, using the simulation data, yields 2.21 for MSA and 2.05 for SA. These results corroborate, in one hand, the suitability of the slip condition and, on the other hand, that the simulation diffusion coefficient value for MSA could be realistic. 
The melting temperature can approximately be determined by analyzing $D$ in terms of the temperature in the range between $200 \mathrm{~K}$ and $400 \mathrm{~K}$ (Fig. 7). It should be noticed that the diffusion is clearly significant from $280 \mathrm{~K}$. This result agree with the experimental melting temperature $^{59,60}$ values $293 \mathrm{~K}$ for MSA and $283 \mathrm{~K}$ for SA. An Arrhenius plot of these results between $300 \mathrm{~K}$ and $400 \mathrm{~K}$ has been displayed in Fig. 8 . The $D$ coefficients clearly verify an Arrhenius behavior:

$$
D(T)=D_{0} \exp \left(-E_{D} / R T\right)
$$

Where $R$ is the molar gas constant, $D_{0}$ the pre-exponential factor (or diffusion at infinite temperature) and $E_{D}$ the activation energy of the diffusion process. The activation energy can be calculated using a least squares procedure, which yields $21.2 \mathrm{~kJ} / \mathrm{mol}$ for MSA and 23.3 $\mathrm{kJ} / \mathrm{mol}$ for SA. The experimental activation energy of SA can be evaluated from the temperature dependence of the shear viscosity. Then, several values, compiled from the Kaye

\& Laby tables book, ${ }^{56}$ have been gathered in Table VI. The temperature dependence can also be described by an Arrhenius function $\eta=\eta_{\infty} \exp \left(E_{A} / R T\right), E_{A}$ being the activation energy and $\eta_{\infty}$ the viscosity at infinite temperature. $E_{A}$ can be calculated using a similar least squares procedure, which yields $21.6 \mathrm{~kJ} / \mathrm{mol}$.

Finally, it should be noted that for MSA, the viscosity coefficient and the $D_{0}$ preexponential factor are very close to the results obtained in our previous work. ${ }^{22}$ It is also important to point out that the procedure employed now is more rigorous because smaller amplitude $\lambda$ values have been used. However, the diffusion coefficient is twice larger than the one computed previously. This discrepancy could probably be attributed to the different charge values of the $\mathrm{CH}_{3}$ group used in both simulations.

\section{Analysis of hydrogen bonds.}

Hydrogen bonding is usually defined using either energetic or geometric criteria. ${ }^{61,62}$ The following geometric definition has been employed: two molecules form a hydrogen bond 
(HB) if the intermolecular oxygen-oxygen distance is smaller than the $g_{O-O}(r)$ first minimum (located at $0.4 \mathrm{~nm}$ ), the intermolecular hydrogen-oxygen distance is smaller than the $g_{H-O}(r)$ first minimum (located at $0.25 \mathrm{~nm}$ ) and the angle between the intermolecular oxygen-oxygen alignment and the intermolecular oxygen-hydrogen bond is smaller than $30^{\circ}$.

The mean number $<n>$ of HB and the percentages $f_{n}$ of molecules with " $n$ " HB have been computed. The results are displayed in Table VII and are compared with those found by Padró et $a l .{ }^{63}$ for methanol and water at the same temperature. Then, every MSA and SA molecule has, on average, $\langle n>=1.7$ and $3.1 \mathrm{HB}$, respectively. Moreover, in case of methanol, the majority of molecules have two HB, denoting a structure basically formed by linear chains of hydrogen bonded molecules. In the case of water, since many molecules have three or four HB, the well-known tetrahedral network of HB is constituted. For MSA the number of molecules with one and two HB is very similar (36 and $43.5 \%$ ). Moreover, an important number of molecules with three HB (14\%) is observed, which could be associated with branching. A representative snapshot, showing a molecule which interacts with other two of its first coordination shell through two HB, has been depicted in Fig. 9 (top). The configuration of linear winding chains of $\mathrm{HB}$ for methanol is a consequence of the existence of one type of oxygens (the oxygen atom of the hydroxyl group) which can act, at the same time, as donors and acceptors. For MSA, and according to the results of $g_{H-O}(r)$ depicted in Fig. 4 (top), the hydrogen bonding acceptors are basically the sulfonyl oxygen atoms (they participate, as acceptors, in the $94 \%$ of all the HB). Therefore, every MSA molecule has two acceptors (the oxygen atoms of the sulfonyl group) and one donor (the oxygen atom of the hydroxyl group). If we compare the methanol and MSA results we can conclude that, probably, the particular molecular acceptor-donor topology of MSA reduces the number of linear chains and, at the same time, increases the number of single HB and, to a lesser extent, the branching. 
SA and water exhibit a similar HB structure which, at the same time, is very different to those of MSA and methanol. Then, more of the $80 \%$ of SA and water molecules are hydrogen bonded to two (22\% for SA and $13.5 \%$ for water), three (35.5\% for SA and $37.5 \%$ for water) and four (26 \% for SA and $42 \%$ for water) molecules. Both systems show a similar network of $\mathrm{HB}$, which is more entangled in case of water because, in one hand, the mean number of HB is larger (3.3 for water and 3.1 for SA) and, on the other hand, the percentage of HB with four molecules is also greater. In case of SA, and according to the $g_{H-O}(r)$ results depicted in Fig. 4 (bottom), the HB acceptors are basically the sulfonyl oxygen atoms (they participate in the $75.5 \%$ of all the HB). Thus, every SA molecule has two acceptors and two donors. Probably, this molecular acceptor-donor distribution increases its capacity to make more complex HB structures, so that the SA network resembles that of water. This behavior can be observed in Fig. 9 (bottom), where a representative snapshot has been depicted, showing a SA molecule interacting with other four of its first coordination shell through four HB.

We have also performed a study of the dynamics of the formation and rupture of HB. Luzar $^{64}$ stated that HB lifetimes can be calculated by two different methodologies, according to what she defines as continuous and interrupted HB lifetimes. The first one has not been considered in this work. The interrupted or slow lifetime, has been calculated by assuming that HB can break and re-form. The probability $c(t)$ that a randomly chosen pair of molecules is bonded at time $t$, provided that it was bonded at $t=0$, is defined. $c(t)$ is computed using the following relation:

$$
c(t)=<h(t) h(0)>/<h(0)^{2}>,
$$

where $h(t)$ is a binary function for each pair of molecules. $h(t)=1$ if molecules " $i, j$ " are bonded at time $t$ and $h(t)=0$ if are not bonded at this time. The MSA and SA $c(t)$ functions at $298 \mathrm{~K}$ have been plotted in Fig. 10 (top). In both cases an exponential behavior, followed by 
a power law tail, is observed. The SA $c(t)$ function evolves to zero faster than the MSA one. In order to quantify this behavior a $\tau_{c}$ lifetime, defined as the $c(t)$ time integral, has been computed. The $\tau_{c}$ lifetimes of both systems are gathered in Table VIII, together with those associated with the HB made with sulfonyl and hydroxyl oxygen acceptors. These results show, in one hand, that the HB made with sulfonyl oxygens, as acceptors, are the most stable (i.e. $\tau_{c}$ are the largest) and, on the other hand, that the MSA lifetime ( $\left.\tau_{c}=22.56 \mathrm{ps}\right)$ is larger than the SA one $\left(\tau_{c}=14.60 \mathrm{ps}\right)$. This last finding is consistent with a recent $a b$ initio computation, made by Bork and coworkers, ${ }^{65}$ of the binding enthalpies of MSA and SA several dimers. According to these authors the values are: $-18.44 \mathrm{kcal} / \mathrm{mol}(\mathrm{SA})$ and -20.28 $\mathrm{kcal} / \mathrm{mol}$ (MSA). This result suggests that the MSA-MSA bonds are stronger than the SA-SA ones.

To complete the description of the HB dynamics, additional time correlation functions, related to the number " $n$ " of HB that a molecule has at a given instant, have been introduced. Particularly, the probability $c_{n}(t)$ that a molecule is in the " $n$ " state, i.e. it has " $n$ " HB, at time $t$, provided that it also has “ $n$ ” $\mathrm{HB}$ at $t=0$, is defined as: ${ }^{66,67}$

$$
c_{n}(t)=<\delta h_{n}(t) \delta h_{n}(0)>/<\delta h_{n}(0)^{2}>,
$$

where $\delta h_{n}(t)=h_{n}(t)-h_{n}$. Now, the binary function $h_{n}(t)=1$ if a molecule is in the " $n$ " HB state at time t, independently of whether or not its HB state has changed in the meantime between 0 and $\mathrm{t} ;(t)=0$, otherwise. $h_{n}$ is the mean value of this function. The lifetimes $\tau_{c n}$ have been calculated as the time integral of every $c_{n}(t)$ function. The corresponding lifetimes for the $n=$ 0,1,2,3,4,5 states are also gathered in Table VIII. To complete the discussion, the lifetimes calculated by Guàrdia et al. ${ }^{67}$, in a recent Car-Parrinello molecular dynamics simulation study of liquid water, have also been included. The $\tau_{c n}$ lifetimes for SA and water are generally more than an order of magnitude smaller than those of MSA. Therefore, as the HB of the former systems break and reform quickly, we can assert that SA and water are more labile 
than MSA. A similar finding has also been reported by Guàrdia et al. ${ }^{67}$ when they compare their results for water with those obtained by Saiz et al. ${ }^{66}$ for liquid ethanol. In case of SA, these findings should be associated to the presence of two HB donors per molecule. Probably, and also for this reason, the SA molecules with three HB are the less stable (the $\tau_{c n}$ lifetimes are the lowest). On the contrary, the presence of one single donor per molecule in case of MSA gives rise to a less labile system, being the most stable molecules those with one and three HB.

The self-diffusion of the system influence $c(t)$ and, therefore, the $\tau_{c}$ value ${ }^{68}$. Particularly, self-diffusion leads to smaller HB relaxation time values. The following correlation function was introduced to minimize this effect: ${ }^{64}$

$$
n(t)=<h(0)[1-h(t)] H(t)>/<h(0)>,
$$

where $H(t)=1$ if the oxygen-oxygen distance between the pair of molecules " $i, j$ " is closer than the first minimum of $g_{O-O}(r)$ at time $t$ and $H(t)=0$ otherwise. Then, the dynamics of the HB is defined using the reactive flux correlation function $k(t)=-d c(t) / d t$, which according to Luzar and Chandler theory, ${ }^{64}$ is related to $n(t)$ and $c(t)$ by the following equation:

$$
k(t)=k c(t)-k^{\prime} n(t)
$$

where $k$ and $k$ ' are the rate constants associated to the processes of break and re-formation of the HB. The MSA and SA $k(t)$ functions have been depicted in Fig. 10 (bottom). Both exhibit a similar behavior, only showing discrepancies in the $k(0)$ value, basically because the SA $c(t)$ decreases faster than the MSA one. At very short times $(t<0.2 \mathrm{ps})$ the dynamics of the HB breaking shows a transient behavior, dominated by librational and inter-oxygen vibrational motions. For larger times $(t>0.2 \mathrm{ps})$ the function decays monotonically, according to the following exponential functional form: $k(t) \sim k e^{-k t}$. The analysis of this decay is generally used to compute the interrupted or slow lifetime, which refers to the time 
constant for HB breakage. It is defined as: $\tau_{L C}=1 / k$. At room temperature the lifetimes values for MSA and SA are respectively $\tau_{L C}=17.20$ ps and $12.67 \mathrm{ps}$.

It is well known that in liquids $\tau_{L C}$ decrease as temperature rises following the next Arrhenius behavior:

$$
\tau_{L C}(T)=\tau_{L C 0} \exp \left(E_{H B} / R T\right),
$$

$\tau_{L C 0}$ being the interrupted $\mathrm{HB}$ lifetime at infinite temperature and $E_{H B}$ the interrupted activation energy. Fig. 11 shows an Arrhenius plot of $\tau_{L C}$ between 300 and $400 \mathrm{~K}$ for both systems. A linear fit yields the following activation energies $E_{H B}=19.6 \mathrm{~kJ} / \mathrm{mol}$ for MSA and $21.7 \mathrm{~kJ} / \mathrm{mol}$ for SA, which are very similar to the activation energies of the self-diffusion 21.2 $\mathrm{kJ} / \mathrm{mol}$ for MSA and $23.3 \mathrm{~kJ} / \mathrm{mol}$ for SA and the experimental activation energy of SA, calculated from the viscosity $21.6 \mathrm{~kJ} / \mathrm{mol}$. As the activation energies of both systems are very similar and the HB network of SA is more labile than that of MSA, the reason because the viscosity of SA is approximately twice that of MSA could be attributed to the larger number of HB around every SA molecule (the mean numbers are 3.1 for SA and 1.7 for MSA). Finally, for MSA the mean number $<n>$ of $\mathrm{HB}$ and the percentages of molecules with " $n$ " HB values are very similar to those obtained in our previous work ${ }^{22}$. However, the lifetimes and the activation energy values of the present work are smaller, probably because of its major diffusivity.

\section{CONCLUSIONS}

Molecular dynamics simulation results of methanesulfonic and sulfuric acids are in a good agreement with some of the available experimental data, such as the density, the shear viscosity and the diffusion coefficients, the melting temperature and the heat of vaporization. The radial distribution functions and the spatial distribution of hydrogen bonds (mean number of hydrogen bonds and percentage of molecules with " $n$ " hydrogen bonds) findings suggest 
that these systems have a different structure of hydrogen bonds. Thus, every sulfuric acid molecule has two acceptors (the oxygen atoms of the sulfonyl group) and two donors (the oxygen atoms of the hydroxyl groups) that give rise to a network of hydrogen bonds, which resembles the one of water. On the contrary, every methanesulfonic acid molecule has one donor (the oxygen atom of the hydroxyl group) and two acceptors (the oxygen atoms of the sulfonyl group), which establishes a hydrogen bond structure, that, in some aspects, recalls the one of methanol, but with an important presence of simple bonds and, to a lesser extent, of branching. The comparison of the interrupted hydrogen bonding lifetimes of both systems shows that the sulfuric acid is more labile (i.e. their hydrogen bonds break and reform quickly) than the methanesulfonic acid. The temperature dependence, between 300 and 400 $\mathrm{K}$, of the diffusion coefficients and the interrupted hydrogen bond lifetimes follow an Arrhenius behavior, with similar activation energies.

\section{ACKNOWLEDGEMENTS}

Financial support from the MINECO and FEDER (FIS2012-39443-C02-01 and FIS201566879-C2-1-P) is gratefully acknowledged. Authors also thank Carlos Alemán for useful suggestions. 


\section{REFERENCES}

${ }^{1}$ M. F. Fingas, Handbook of Hazardous Materials Spills Technology (McGraw-Hill, New York, 2002).

${ }^{2}$ S. Patai and Z. Rappoport, The Chemistry of Sulphonic Acids, Esters and their Derivatives (John Wiley \& Sons, New York, 1991).

${ }^{3}$ M. D. Gernon, M. Wu, T. Buzsta and P. Janney, Green Chem. 1, 127 (1999).

${ }^{4}$ T. A. Skotheim, R. L. Elsenbaumer and J. R. Reynolds, Handbook of Conducting Polymers (Marcel Dekker, New York, 1998).

${ }^{5}$ M. L. Dawson, M. E. Varner, V. Perraud, M. J. Ezell, R. B. Gerber and B. J. Finlayson-Pitts, PNAS 109, 18719 (2012).

${ }^{6}$ R. J. Charlson, J. E. Lovelock, M. O. Andreae and S. G. Warren, Nature 326, 655 (1987).

${ }^{7}$ S. C. Baker, D. P. Kelly and J. C. Murrell, Nature 350, 627 (1991)

${ }^{8}$ H. Arstila, K. Laasonen and A. Laaksonen, J. Chem. Phys. 108, 1031 (1998).

${ }^{9}$ S. M. Kathmann and B. N. Hale, J. Phys. Chem. B 105, 11719 (2001).

${ }^{10}$ I. Kusaka, Z. G. Wang and J. H. Seinfeld, J. Chem. Phys. 108, 6829 (1998).

${ }^{11}$ C. Ding, T. Taskila, K. Laasonen and A. Laaksonen, Chem. Phys. 287,7 (2003).

${ }^{12}$ M. Toivola, I. Napari and H. Vehkamäki, Boreal Environment Research 14, 654 (2009).

${ }^{13}$ H. Matsubara, T. Ebisuzaki and K. Yasuoka, J. Chem. Phys. 130, 104705 (2009).

${ }^{14}$ K. Choe, E. Tsuchida and T. Ikeshoji, J. Chem. Phys. 126, 154510 (2007).

${ }^{15}$ T. Ishiyama and A. Morita, J. Phys. Chem. C 115, 13704 (2011).

${ }^{16}$ J. R. Durig, L. Zhou, T. Schwartz and T. Gounev, J. Raman Spectros. 31, 193 (2000).

${ }^{17}$ L. Wang and J. Zhang, J. Mol. Struct.: TEOCHEM. 581, 129 (2002).

${ }^{18}$ N. F. Carvalho, S. P. Silva and S. M. Resende, J. Braz. Chem. Soc. 22, 950 (2011).

${ }^{19}$ L. Wang, J. Phys. Chem. A 111, 3642 (2007).

${ }^{20}$ A. Krishtal, P. Senet and C. van Alsenoy, J. Chem. Theory Comput. 4, 2122 (2008). 
${ }^{21}$ A. Givan, A. Loewenschuss and C. J. Nielsen, J. Mol. Struct. 748, 77 (2005).

${ }^{22}$ M. Canales and C. Aleman, J. Phys. Chem. B 118, 3423 (2014).

${ }^{23}$ X. Chen, B. Minofar, P. Jungwirth and H. C. Allen, J. Phys. Chem. B 114, 15546 (2010).

${ }^{24}$ G. A. Jeffrey, An Introduction to Hydrogen Bonding (Oxford University Press, New York, 1997).

${ }^{25}$ S. J. Grabowski (ed), Hydrogen Bonding-New Insights (Springer, Dordrecht, 2006).

${ }^{26}$ B. M. Ladanyi and M. S. Skaf, Annu. Rev. Phys. Chem. 44, 335 (1993).

${ }^{27}$ Y. Marechal, The Hydrogen Bond and the Water Molecule: The Physics and Chemistry of Water, Aqueous and Bio-Media (Elsevier, Amsterdam, 2007).

${ }^{28}$ M. Matsumoto, K. E. Gubbins, J. Chem. Phys. 93, 1981 (1990).

${ }^{29}$ Gaussian 03, Revision C.02, M. J. Frisch, G. W. Trucks, H. B. Schlegel, G. E. Scuseria, M. A. Robb, J. R. Cheeseman, J. A. Montgomery, Jr., T. Vreven, K. N. Kudin, J. C. Burant, J. M. Millam, S. S. Iyengar, J. Tomasi, V. Barone, B. Mennucci, M. Cossi, G. Scalmani, N. Rega, G. A. Petersson, H. Nakatsuji, M. Hada, M. Ehara, K. Toyota, R. Fukuda, J. Hasegawa, M. Ishida, T. Nakajima, Y. Honda, O. Kitao, H. Nakai, M. Klene, X. Li, J. E. Knox, H. P. Hratchian, J. B. Cross, V. Bakken, C. Adamo, J. Jaramillo, R. Gomperts, R. E. Stratmann, O. Yazyev, A. J. Austin, R. Cammi, C. Pomelli, J. W. Ochterski, P. Y. Ayala, K. Morokuma, G. A. Voth, P. Salvador, J. J. Dannenberg, V. G. Zakrzewski, S. Dapprich, A. D. Daniels, M. C. Strain, O. Farkas, D. K. Malick, A. D. Rabuck, K. Raghavachari, J. B. Foresman, J. V. Ortiz, Q. Cui, A. G. Baboul, S. Clifford, J. Cioslowski, B. B. Stefanov, G. Liu, A. Liashenko, P. Piskorz, I. Komaromi, R. L. Martin, D. J. Fox, T. Keith, M. A. Al-Laham, C. Y. Peng, A. Nanayakkara, M. Challacombe, P. M. W. Gill, B. Johnson, W. Chen, M. W. Wong, C. Gonzalez, and J. A. Pople, Gaussian, Inc., Wallingford CT, 2004.

${ }^{30}$ A. D. Becke, J. Chem. Phys. 98, 1372 (1993).

${ }^{31}$ C. Lee, W. Yang and R. G. Parr, Phys. Rev. B 37, 785 (1988). 
${ }^{32}$ U. C. Singh and P. A. Kollman, J. Comput. Chem. 5, 129 (1984).

${ }^{33}$ B. H. Besler, K. Merz and P. A. Kollman, J. Comput. Chem. 11, 431 (1990).

${ }^{34}$ V. Loukonen, T. Kurtén, I. K. Ortega, H. Vehkamäki, A. A. H. Pádua, K. Sellegri and M. Kulmala, Atmos. Chem. Phys. 10, 4961 (2010).

${ }^{35}$ B. Hess, C. Kutzner and D. van der Spoel, J. Chem. Theory Comput. 4, 435 (2008).

${ }^{36}$ J. P. Ryckaert, G. Cicotti and H. J. C. Berendsen, J. Comput. Phys. 23, 327 (1977).

${ }^{37}$ W. L. Jorgensen, D. S. Maxwell and J. Tirado-Rives, J. Am. Chem. Soc. 118, 11225 (1996).

${ }^{38}$ M. H. Hetko, G. Kamath and J. J. Potoff, J. Phys. Chem. B 115, 4949 (2011).

${ }^{39}$ J. P. Ryckaert and A. Bellemans, Chem. Phys. Lett. 30, 123 (1975).

${ }^{40}$ S. J. Paddison, J. New. Mat. Eletrochem. Systems 4, 197 (2001).

${ }^{41}$ C. Oostenbrink, A. Villa, A. E. Mark and W. F. van Gunsteren, J. Comput. Chem. 25, 1656 (2004).

${ }^{42}$ H. J. C. Berendsen, J. P. M. Postma, W. F. van Gunsteren, A. DiNola and J. R. Haak, J. Chem. Phys. 81, 3684 (1984).

${ }^{43}$ M. P. Allen and D. J. Tildesley, Computer Simulation of Liquids (Oxford University Press, New York, 1987).

${ }^{44}$ T. Darden, D. York and L. Pedersen, J. Chem. Phys. 98, 10089 (1993).

${ }^{45}$ A. R. Leach, Molecular Modelling: Principles and Applications (Pearson, Harlow, 2001).

${ }^{46}$ R. L. Kuczkowski, R. D. Suenram and F. J. Lovas, J. Am. Chem. Soc. 103, 2561 (1981).

${ }^{47}$ A. Givan, L. A. Larsen, A. Loewenschuss and C. J. Nielsen, J. Mol. Struct. 509, 35 (1999).

${ }^{48}$ D. B. Roitman, J. McAlister and F. L. Oaks, J. Chem. Eng. Data 39, 56 (1994).

${ }^{49}$ R. H. Perry, D. W. Green and J. O. Maloney, Perry's Chemical Engineers' Handbook $7^{\text {th }}$ ed. (McGraw-Hill, New York, 1997).

${ }^{50}$ Tables of Physical and Chemical Constants (16 ${ }^{\text {th }}$ edition 1995). 2.3.4 Thermal expansion. Kaye \& Laby Online. Version 1.1 (2010) www.kayelaby.npl.co.uk 
${ }^{51}$ C. Andreani, C. Petrillo and F. Sachetti, Mol. Phys. 58, 299 (1986).

${ }^{52}$ V. Kazansky and V. Solkan, Phys. Chem. Chem. Phys. 5, 31 (2003).

${ }^{53}$ C. Caleman, P. J. van Maaren, M. Hong, J. S. Hub, L. T. Costa and D. van der Spoel, J. Chem. Theory Comput. 8, 61 (2012).

${ }^{54}$ J. P. Guthrie, A. R. Stein and A. P. Huntington, Can. J. Chem. 76, 929 (1998).

${ }^{55}$ B. Hess, J. Chem Phys. 116, 209 (2002).

${ }^{56}$ Tables of Physical and Chemical Constants (16 ${ }^{\text {th }}$ edition 1995). 2.2.3 Viscosities. Kaye \& Laby Online. Version 1.0 (2005) www.kayelaby.npl.co.uk

${ }^{57}$ K. R. Harris, J. Chem. Soc., Faraday Trans. 1 78, 1629 (1982).

${ }^{58}$ J. P. Hansen and I. R. McDonald, Theory of Simple Liquids (Academic Press, Amsterdam, 2006).

${ }^{59}$ Merck Index, An Encyclopedia of Chemical, Drugs and Biologicals (Merck, Whitehouse Station NJ, 2006).

${ }^{60}$ D. R. Lide, CRC Handbook of Chemistry and Physics, $88^{\text {th }}$ Edition (CRC Press, Boca Raton, FL, 2007).

${ }^{61}$ D. Bertolini, M. Cassettari, M. Ferrario, P Grigolini and G. Salvetti, Adv. Chem. Phys.62, 277 (1985).

${ }^{62}$ F. Sciortino and S. L. Fornili, J. Chem. Phys. 90, 2786 (1989).

${ }^{63}$ J. A. Padró, L. Saiz and E. Guàrdia, J. Molec. Struct. 416, 243 (1997).

${ }^{64}$ A. Luzar, J. Chem. Phys. 113, 10663 (2000).

${ }^{65}$ N. Bork, J. Elm, T. Olenius and H. Vehkamäki, Atmos. Chem. Phys. 14, 12023 (2014).

${ }^{66}$ L. Saiz, J. A. Padró and E. Guàrdia, Mol. Phys. 97, 897 (1999).

${ }^{67}$ E. Guàrdia, I. Skarmoutsos and M. Masia, J. Phys. Chem. B 119, 8926 (2015).

${ }^{68}$ H. S. Lee and M. E. Tuckerman, J. Chem. Phys. 126, 164501 (2007). 


\section{TABLES}

TABLE I: Partial charges (atomic units) of the SA and MSA atoms computed in this work and those of references 8, 11 and 34 .

\begin{tabular}{cccccc}
\hline \hline Atom & MSA & SA & SA (ref 8) & SA (ref 11) & SA (ref 34) \\
\hline \hline H & 0.202 & - & - & - & - \\
C & -0.520 & - & - & - & - \\
S & 0.960 & 1.000 & 0.81 & 0.868 & 1.100 \\
O & -0.468 & -0.420 & -0.37 & -0.397 & -0.460 \\
O' & -0.500 & -0.480 & -0.49 & -0.480 & -0.530 \\
H' & 0.390 & 0.415 & 0.45 & 0.433 & 0.440 \\
\hline \hline
\end{tabular}

TABLE II: Bond lengths (nm).

\begin{tabular}{ccc}
\hline \hline Bond & MSA & SA \\
\hline \hline S-C & 0.1788 & - \\
S-O' & 0.1631 & 0.1647 \\
S=O & 0.1446 & 0.1466 \\
O’-H’ & 0.0968 & 0.0973 \\
C-H & 0.1086 & - \\
\hline \hline
\end{tabular}


TABLE III: Bond angles (degrees) and harmonic force constants $\left(\mathrm{kJmol}^{-1}\right)$.

\begin{tabular}{|c|c|c|c|}
\hline \multirow[t]{2}{*}{ Bond } & \multicolumn{2}{|c|}{ Angle } & \multirow[t]{2}{*}{$K_{\theta}$} \\
\hline & MSA & SA & \\
\hline $\mathrm{H}^{\prime}-\mathrm{O}^{\prime}-\mathrm{S}$ & 107.5 & 107.4 & 185 \\
\hline $\mathrm{O}^{\prime}-\mathrm{S}-\mathrm{O}^{\prime}$ & - & 101.5 & 195 \\
\hline O'-S-O & 107.1 & 107.0 & 200 \\
\hline $\mathrm{O}-\mathrm{S}-\mathrm{O}$ & 121.4 & 125 & 230 \\
\hline O'-S-C & 100.6 & - & 220 \\
\hline O-S-C & 109.3 & - & 220 \\
\hline S-C-H & 107.8 & - & 146 \\
\hline H-C-H & 111.1 & - & 138 \\
\hline
\end{tabular}

TABLE IV: Ryckaert-Bellemans torsional potential parameters in $\mathrm{kJmol}^{-1}$.

\begin{tabular}{ccccccc}
\hline \hline Dihedral & C0 & C1 & C2 & C3 & C4 & C5 \\
\hline \hline H-C-S-O,O' & 0.9414 & 2.8242 & 0 & -3.7656 & 0 & 0 \\
H'-O'-S-O,C & 1.8 & -5.4 & 0 & 7.2 & 0 & 0 \\
H'-O'-S-O,O' & 1.8 & -5.4 & 0 & 7.2 & 0 & 0 \\
\hline \hline
\end{tabular}


TABLE V: Lennard-Jones parameters ( $\sigma$ in $\mathrm{nm}$ and $\varepsilon$ in $\mathrm{kJmol}^{-1}$ ) and partial charges (atomic units).

\begin{tabular}{ccccc}
\hline \hline Atom & $\sigma$ & $\varepsilon$ & $q$ (MSA) & $q(\mathrm{SA})$ \\
\hline \hline $\mathrm{H}$ & 0.250 & 0.1255 & 0.17 & - \\
$\mathrm{C}$ & 0.350 & 0.2761 & -0.51 & - \\
$\mathrm{S}$ & 0.355 & 1.0465 & 0.85 & 0.82 \\
$\mathrm{O}$ & 0.296 & 0.8786 & -0.405 & -0.375 \\
$\mathrm{O}$, & 0.312 & 0.7113 & -0.49 & -0.45 \\
$\mathrm{H}$ & 0 & 0 & 0.45 & 0.415 \\
\hline \hline
\end{tabular}

TABLE VI: Experimental viscosities (cP) of pure sulfuric acid at several temperatures (K), compiled from the Kaye and Laby tables book. ${ }^{56}$

\begin{tabular}{cc}
\hline \hline Temperature & Viscosity \\
\hline \hline 298 & 23.8 \\
303 & 20.1 \\
323 & 11.7 \\
348 & 6.6 \\
373 & 4.1 \\
\hline \hline
\end{tabular}


TABLE VII: Percentages of molecules with " $n$ " hydrogen bonds and mean number of hydrogen bonds for different systems at room temperature.

\begin{tabular}{ccccccccc}
\hline \hline System & $f_{0}$ & $f_{1}$ & $f_{2}$ & $f_{3}$ & $f_{4}$ & $f_{5}$ & $f_{6}$ & $<n>$ \\
\hline \hline MSA & 5.5 & 36 & 43.5 & 14 & 1 & 0 & 0 & 1.7 \\
SA & 1 & 6.5 & 22 & 35.5 & 26 & 8 & 1 & 3.1 \\
Methanol & 1 & 16.5 & 75.5 & 7 & 0 & 0 & 0 & 1.9 \\
Water & 0 & 2 & 13.5 & 37.5 & 42 & 5 & 0 & 3.3 \\
\hline \hline
\end{tabular}

TABLE VIII: Hydrogen bonds lifetimes for MSA and SA at room temperature in ps. $\tau_{c}$ are the ones computed from the integral of $c(t), \tau_{c s}$ and $\tau_{c h}$ their sulfonyl and hydroxyl group contributions, $\tau_{c n}$ those associated to molecules hydrogen bonded to other $n$ molecules and $\tau_{L C}$ the ones computed from the Luzar and Chandler theory. Water results have been compiled from reference 67.

\begin{tabular}{lcccccccccc}
\hline \hline System & $\tau_{c}$ & $\tau_{c s}$ & $\tau_{c h}$ & $\tau_{c 0}$ & $\tau_{c 1}$ & $\tau_{c 2}$ & $\tau_{c 3}$ & $\tau_{c 4}$ & $\tau_{c 5}$ & $\tau_{L C}$ \\
\hline \hline MSA & 22.56 & 23.60 & 4.04 & 1.69 & 6.70 & 3.38 & 7.59 & 2.24 & - & 17.20 \\
SA & 14.60 & 17.10 & 6.60 & 0.11 & 0.61 & 0.69 & 0.16 & 0.76 & 1.00 & 12.67 \\
Water & 4.90 & - & - & 0.03 & 0.08 & 0.19 & 0.15 & 0.24 & 0.17 & - \\
& & & & & & & & & & \\
\hline \hline
\end{tabular}




\section{CAPTIONS TO FIGURES}

FIG. 1. Most favorable structures of MSA and SA molecules. Labels correspond to the atom types defined in Tables I and V.

FIG. 2. Densities of MSA and SA in the range of temperatures between $200 \mathrm{~K}$ and $400 \mathrm{~K}$.

FIG. 3. Center of mass-center of mass radial distribution functions $g_{C M-C M}(r)$ (top) and orientational correlation functions $<\cos \theta(\mathrm{r})>$ (bottom) at $200 \mathrm{~K}, 298 \mathrm{~K}$ and $400 \mathrm{~K}$. MSA (left) and SA (right).

FIG. 4. Hydrogen-oxygen radial distribution function $g_{H O}(r)$ and hydroxyl and sulfonyl group contributions for MSA (top) and SA(bottom) at $298 \mathrm{~K}$.

FIG. 5. Hydrogen-oxygen radial distribution function $g_{H O}(r)$ for SA at $200 \mathrm{~K}, 298 \mathrm{~K}$ and 400 K (top). Oxygen-oxygen radial distribution function $g_{O O}(r)$ for SA and MSA at $298 \mathrm{~K}$ (bottom).

FIG. 6. Shear viscosities of MSA and SA at $298 \mathrm{~K}$ for different acceleration amplitudes.

FIG. 7. Self-diffusion coefficients of MSA and SA in the range of temperatures between 200 $\mathrm{K}$ and $400 \mathrm{~K}$.

FIG. 8. Arrhenius plot of the temperature dependence of the self-diffusion coefficients of MSA and SA between $300 \mathrm{~K}$ and $400 \mathrm{~K}$.

FIG. 9. Representative snapshots of hydrogen bonded molecules. Top: MSA molecule bonded to other two by two hydrogen bonds. Bottom: SA molecule bonded to other four by four hydrogen bonds.

FIG. 10. $c(t)$ probability function and reactive flux correlation function $k(t)$ of MSA and SA at $298 \mathrm{~K}$.

FIG. 11. Arrhenius plot of the temperature dependence of the interrupted hydrogen bond lifetimes of MSA and SA, calculated from the Luzar and Chandler theory, between 300 and $400 \mathrm{~K}$. 


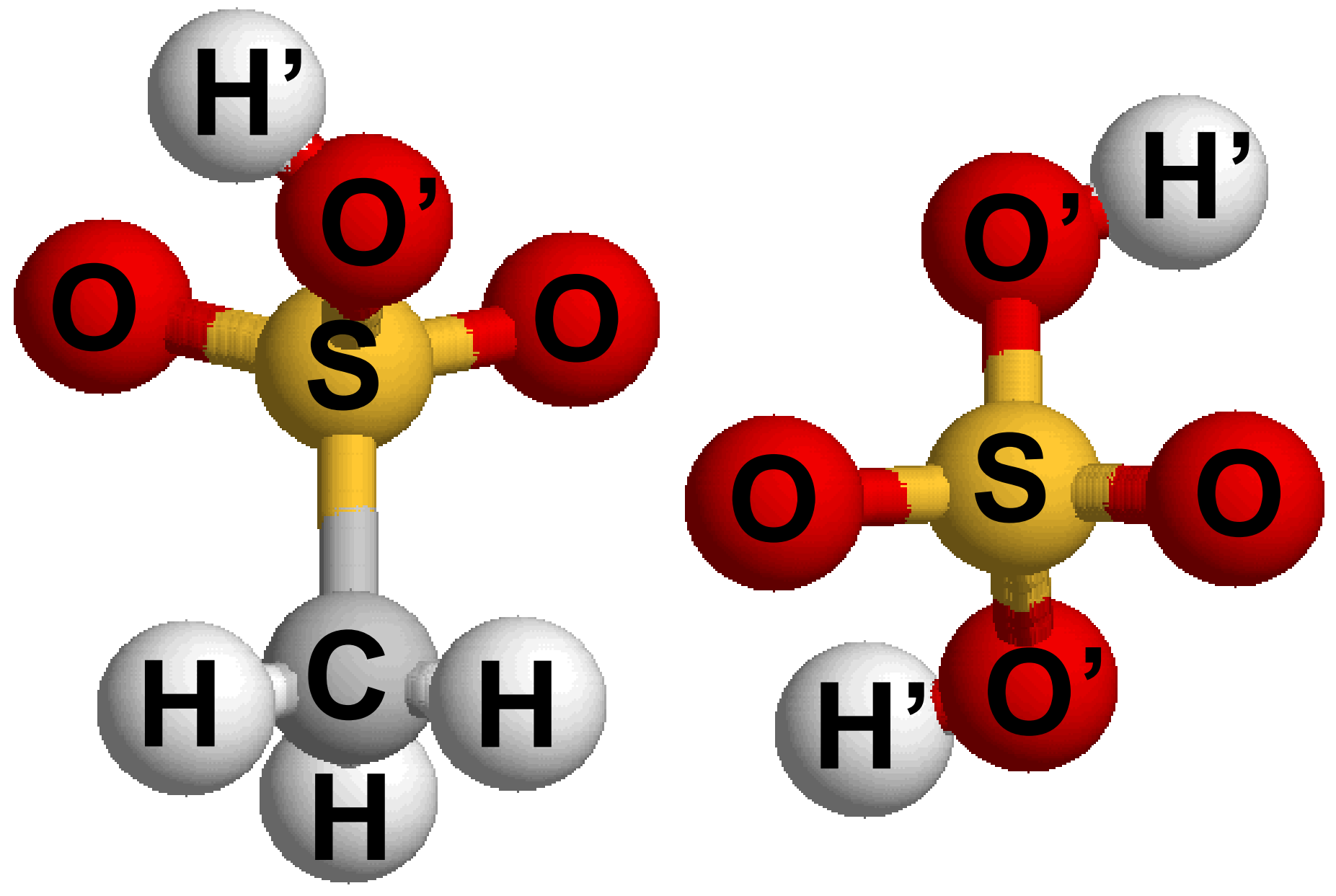

Figure 1 


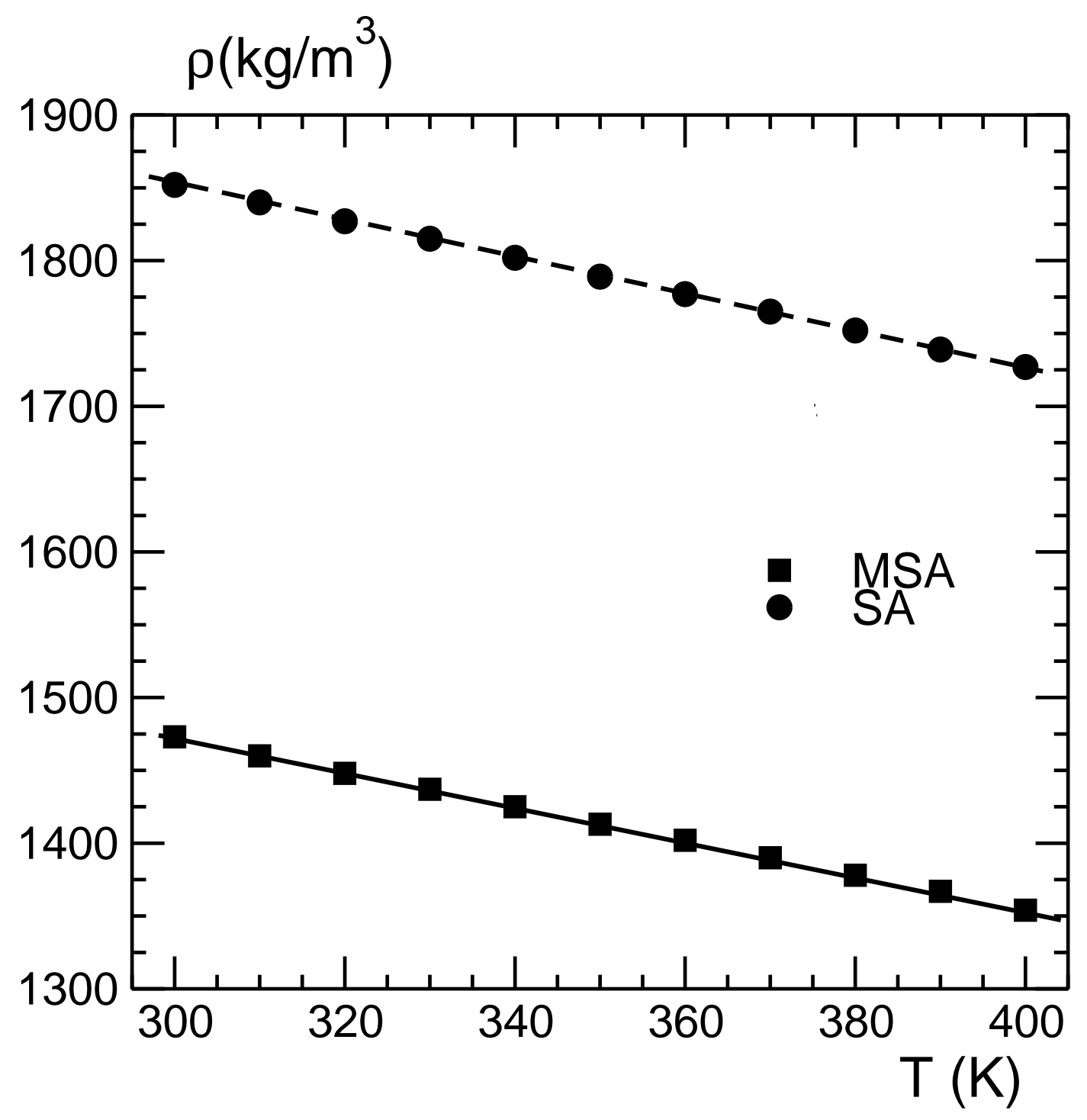

Figure 2 


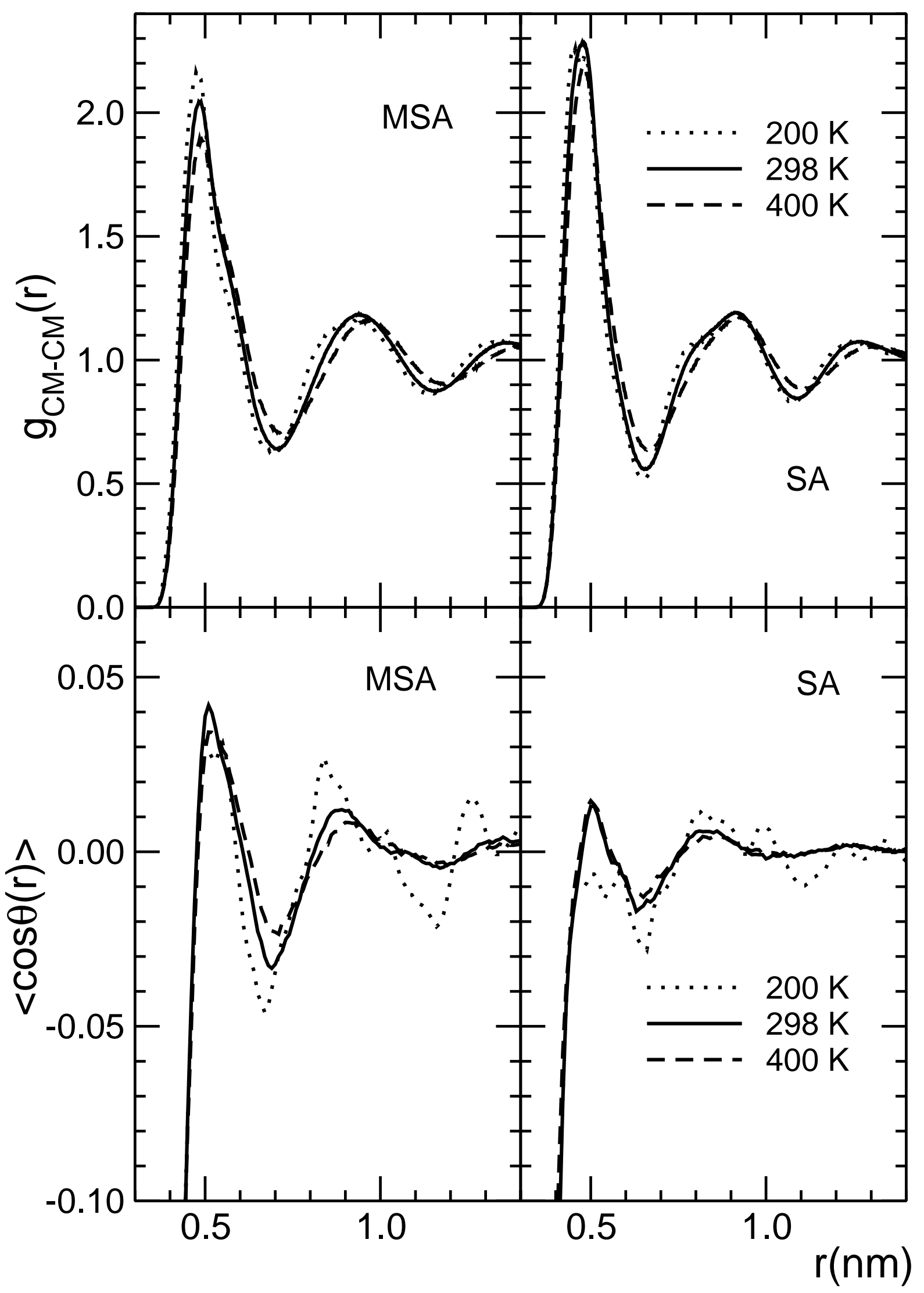

Figure 3 


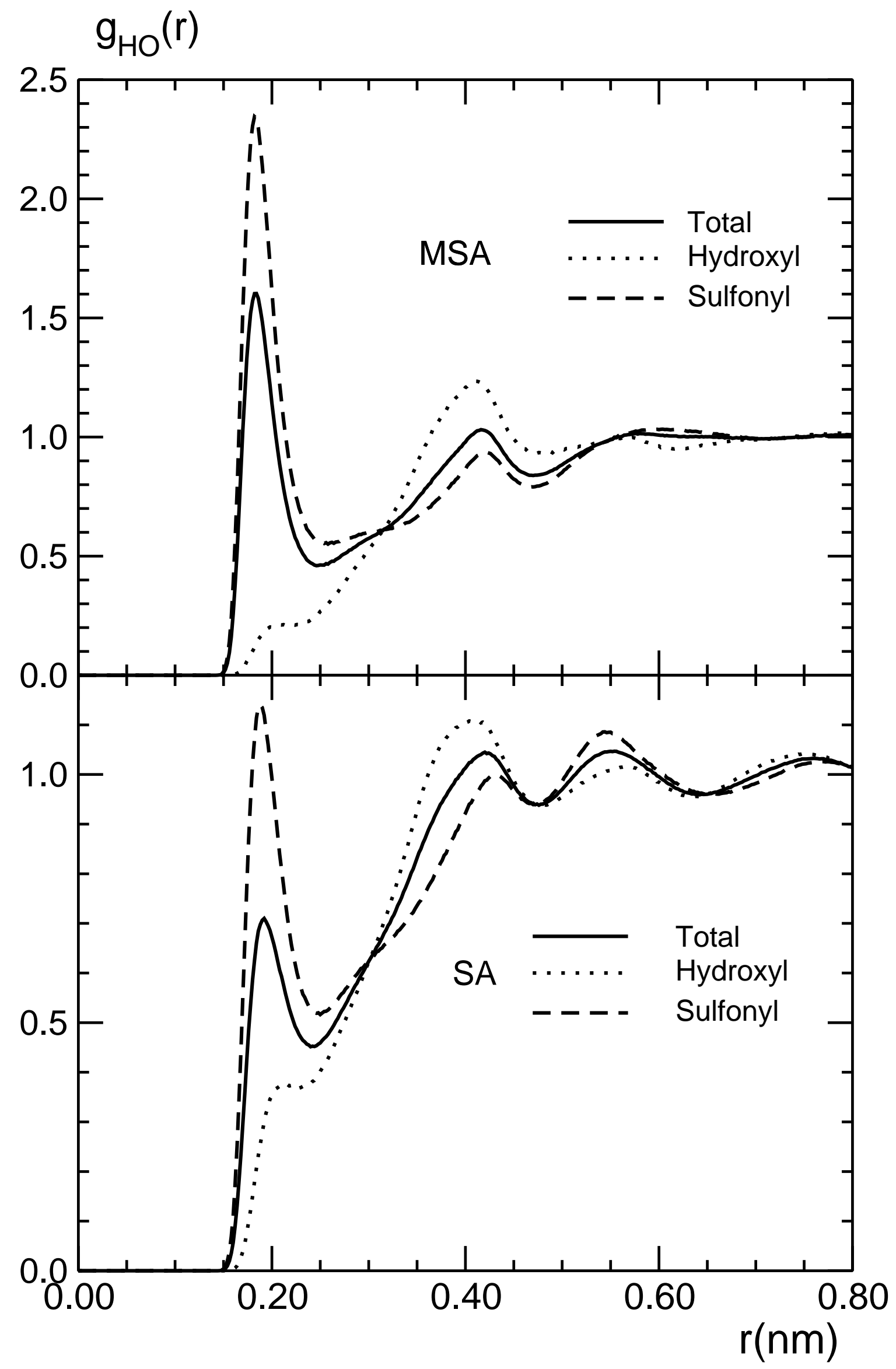

Figure 4 


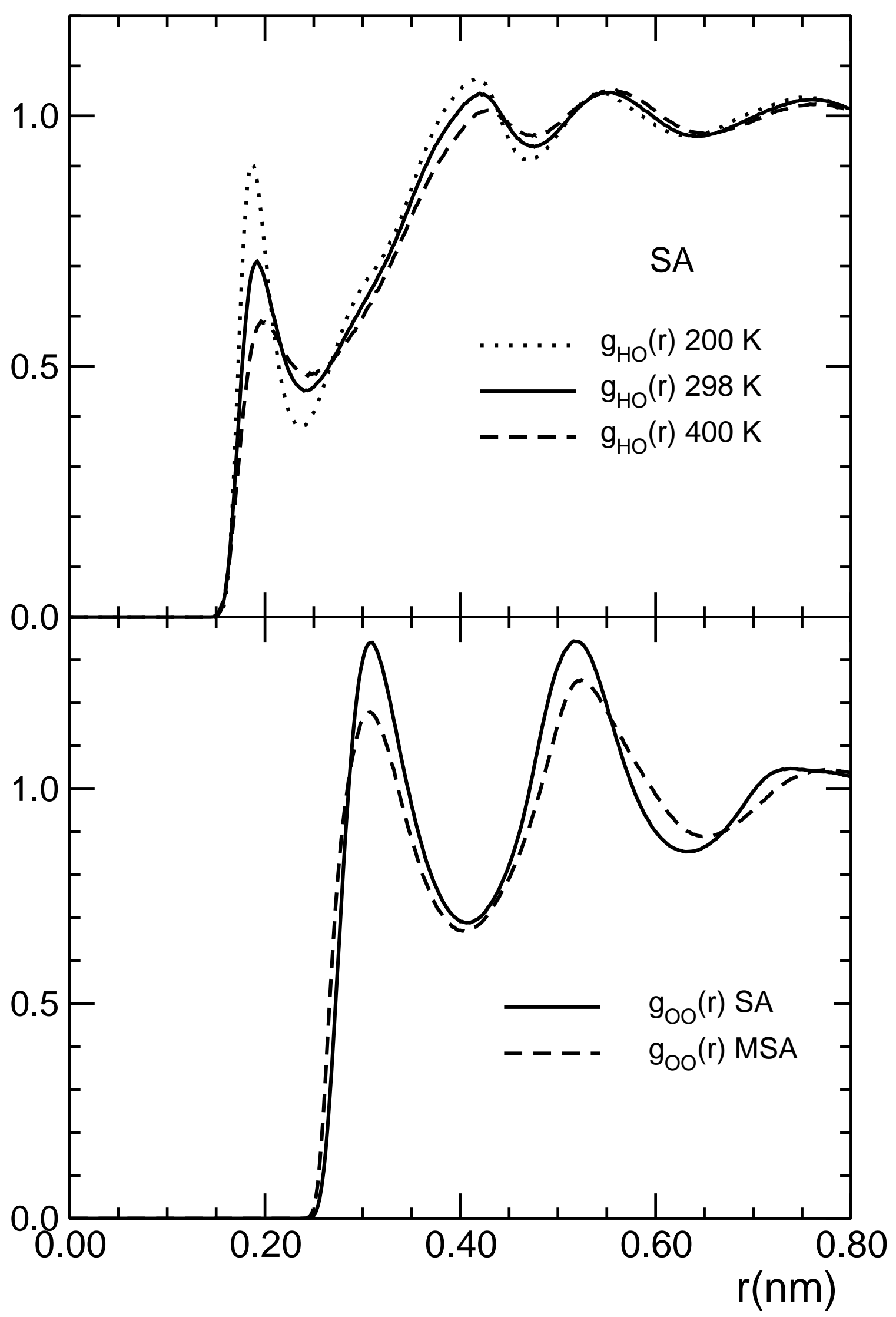

Figure 5 


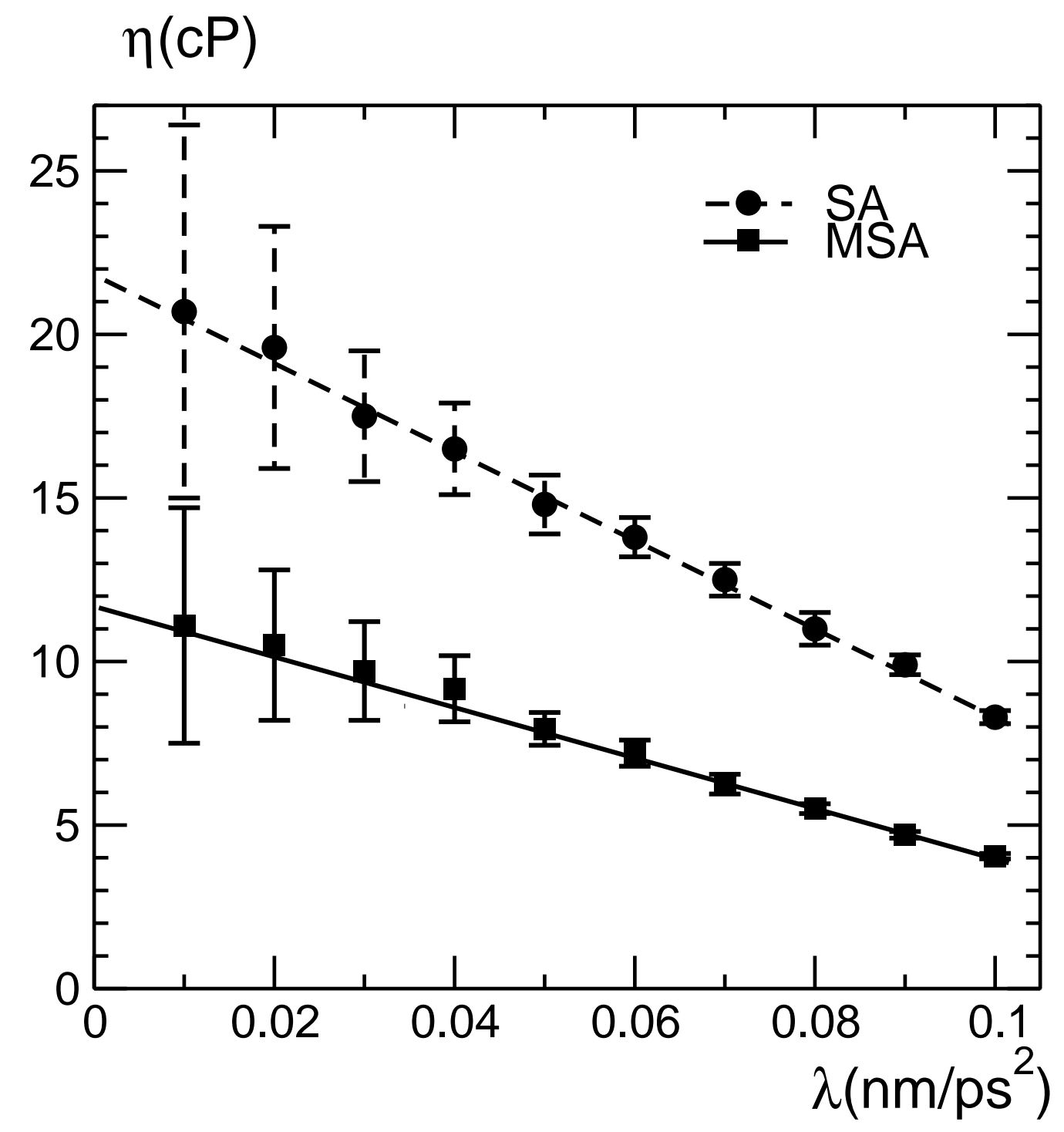

Figure 6 


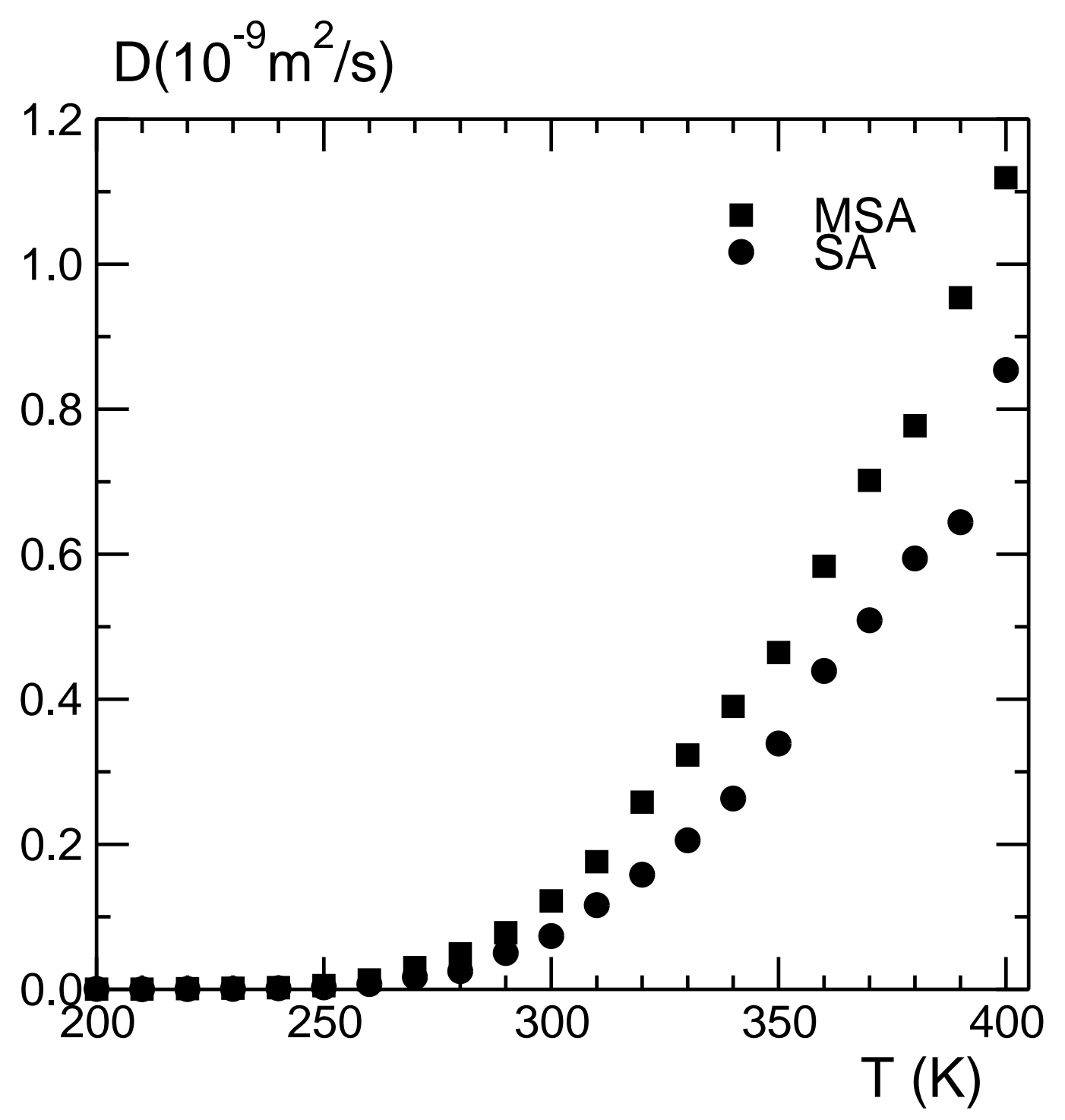

Figure 7 


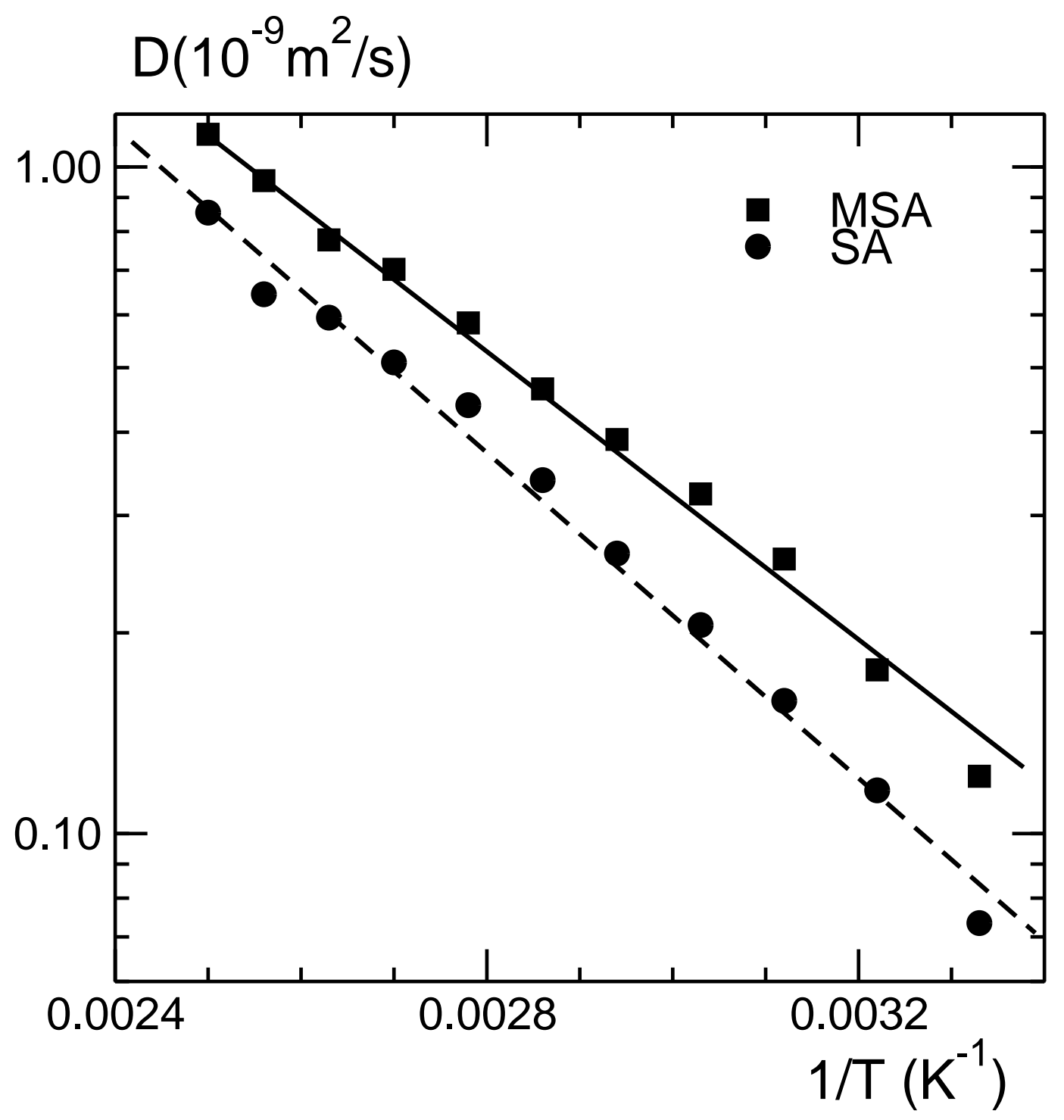

Figure 8 

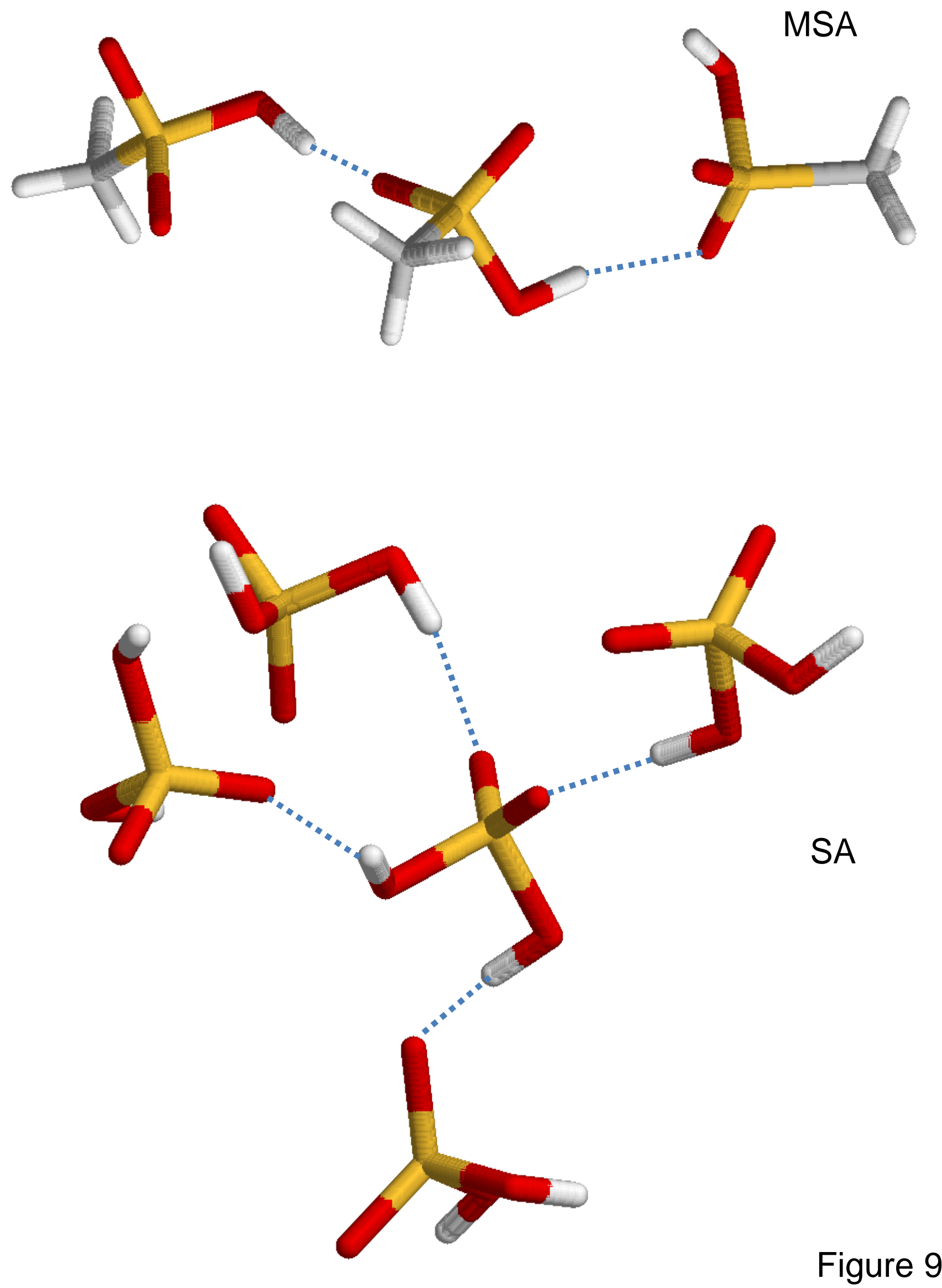

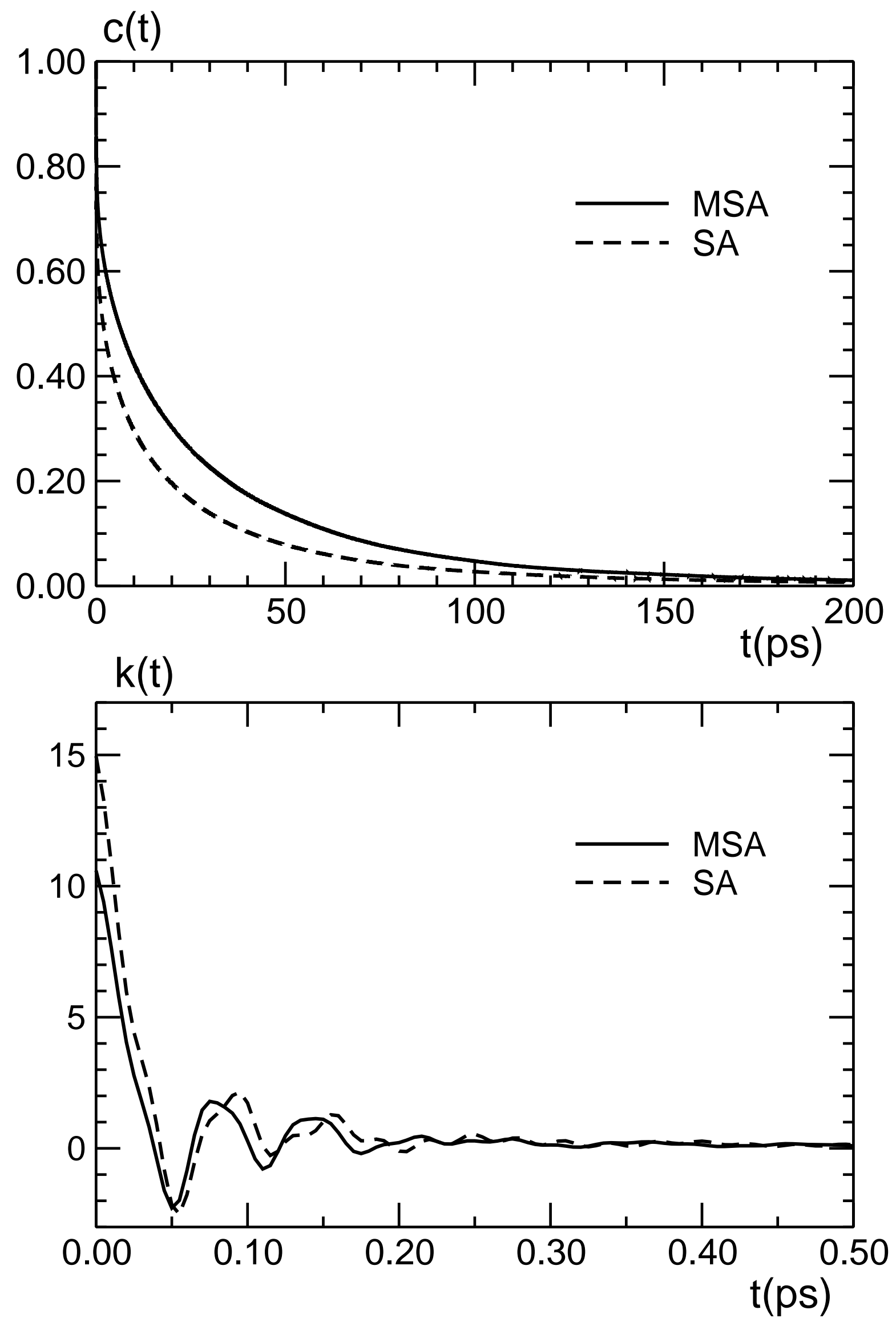

Figure 10 


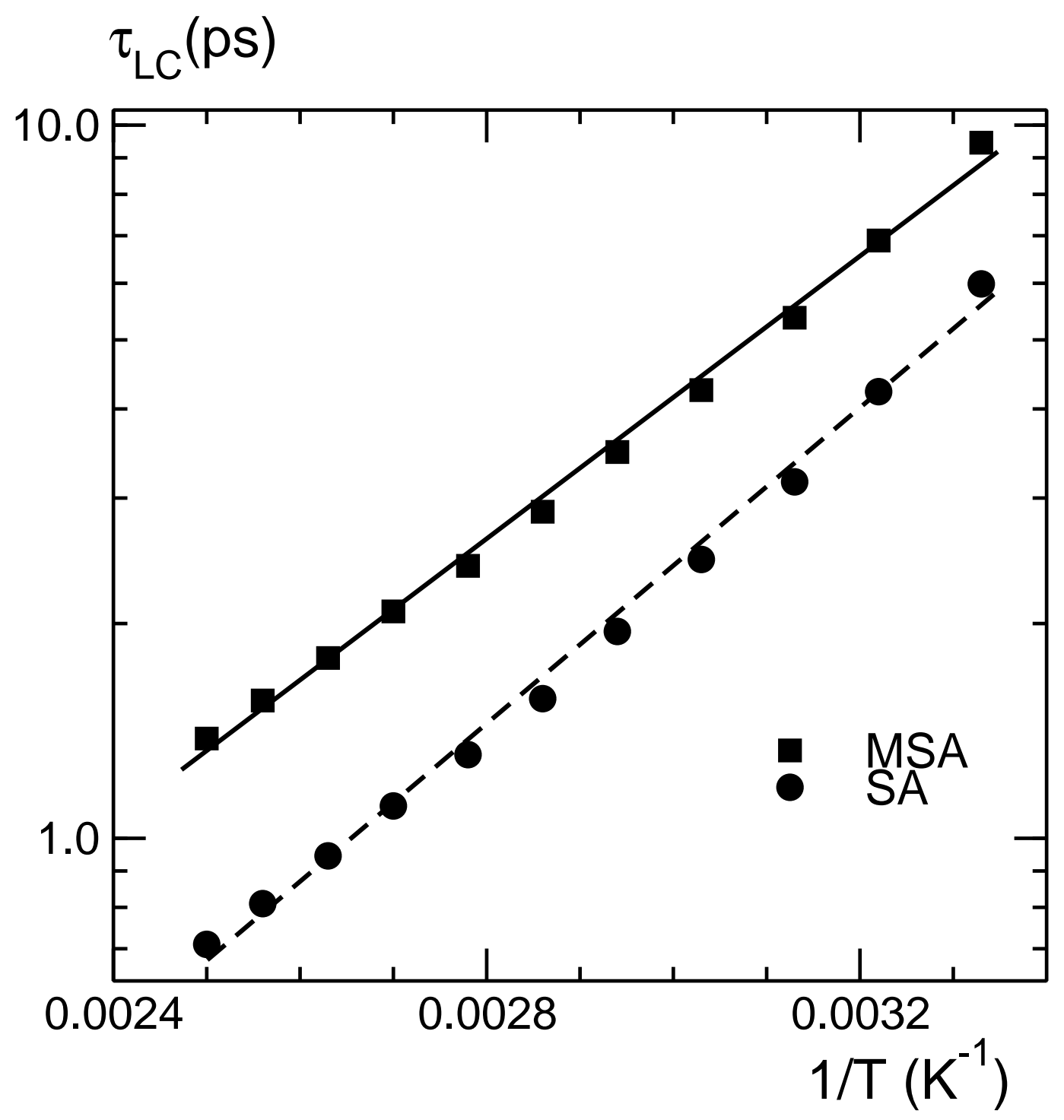

Figure 11 